\title{
Review of Health Information Systems in Oman
}

\author{
Mohammed Elhadi*, Ahmed Al-Hosni*, Khaled Day*, Adbullah Al-Hamadani*, , \\ Abdulrahman Al-Toqi**, Nasser Al-Shamli** and Ali Al-Hashmi***.
}

${ }^{*}$ Department of Computer Science, College of Science, Sultan Qaboos University, P.O.Box 36, PC 123, Al-Khodh, Sultanate of Oman, Muscat, *Email: elhadi@squ.edu.om, ** Directorate General of Information Technology, Ministry of Health, Muscat, Oman, ${ }^{* * * H o s p i t a l}$ Information System Department, Sultan Qaboos University Hospital, Alkhod, Oman.

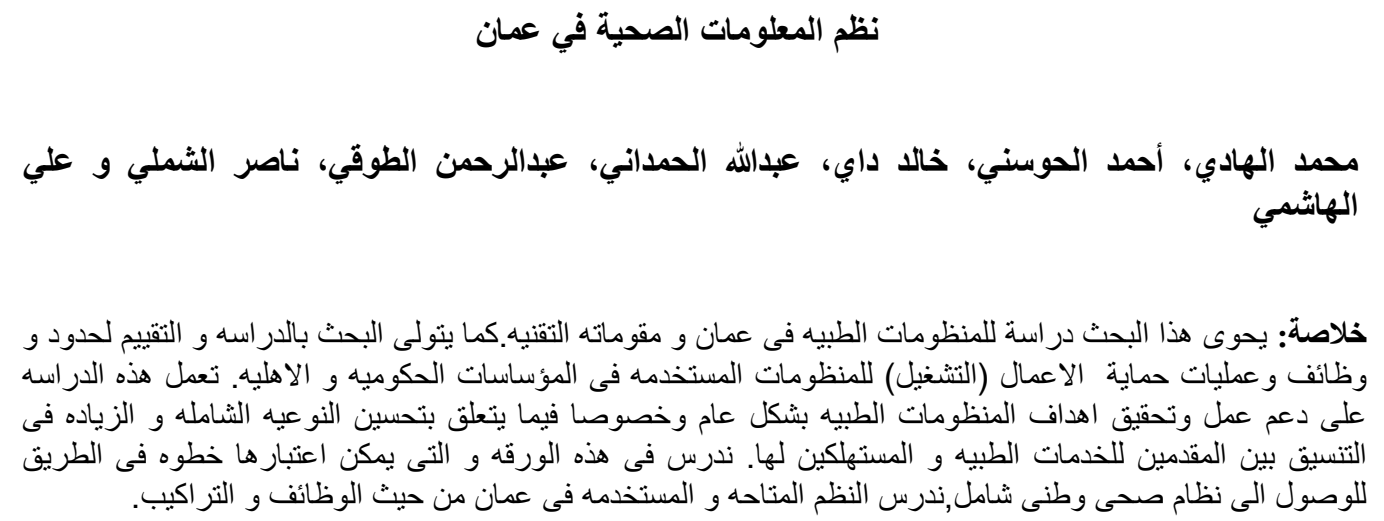

\begin{abstract}
This paper is a review of Oman's major Health Information Systems (HISs) and their enabling technologies. The work assesses the scope, functionality, security, and interoperability of the used systems. The review aids in achieving the objectives of HIS systems of improving the global quality of health care, attaining increased coordination between health care providers and consumers, promoting the use of guidelines and policies, and improving the speed of simultaneous access and distribution of medical records and other resources. This paper, which can be considered as a building block towards the development of a nation-wide health care system, studies existing systems and identifies their functions and structures.
\end{abstract}

KEYWORDS: Health information systems; oman; heath care; information technology.

\section{Introduction}

$\mathbf{P}$ atient-centered and information-rich health care systems, in which medical information follows the patient, and information tools guide medical decisions are aspired for by every health care provider and consumer. Health Information Technology (HIT) is an enabling technology that encompasses various hardware and software systems used for storing, processing and exchanging medical information. Such systems promise to 


\section{MOHAMMED ELHADI, ET AL}

have the potential of transforming traditional health care delivery by bringing information when it is needed and where it is needed; thereby, improving the quality of health care and reducing the cost of delivery (Ortiz and Clancy, 2003) (USA HHS, 2004) (CHT and IDX Systems Corporation, 2005).

Numerous health care related software systems are in use these days under various names such as Computer Patient Record (CPR), Patient Medical Record (PMR), Computerized Medical Record (CMR), Electronic Patient Record (EPR), Electronic Medical Record (EMR), Digital Medical Record (DMR), Patient Medical Record Information (PMRI), Personal Health Record (PHR), Electronic Health Record (EHR), Picture Archiving and Communication (PACS), Medical Diagnostic Imaging Support (MDIS) and many more (WHO, 2004). All of these are just different ways of referring to similar if not the same entity representing the core of information about patients. We will be using the EHR as our reference in this paper.

This article looks at the current status of Health Information Systems (HIS), their enabling technologies and similar products and services that exist in Oman. The work will assess the scope, functionality, security, and interoperability of the considered systems.

Work done in this direction will aid in achieving the objectives of HIS systems of improving global quality of the health care through the computerized support of various functions. This work will also help in improving all of the basic functions of HIS such as scheduling, patient information capturing and retrieval, diagnosis decision support, protocol consultation, care plan management, drug management, scheduling for surgery processing, patient education, quality assurance, and financial transactions.

This work will also help in attaining increased coordination between different health care providers and consumers, promote the use of guidelines and policies, and improve the speed of simultaneous access and free distribution of medical records and resources.

Currently, all the major public health institutions in Oman are computerized; that includes all the national referral hospitals, regional referral hospitals, polyclinics and extended health centers, and all the health centers built after 1995 .

Therefore, it is important to integrate these individual information systems into a connected nation-wide system that provides secure coordinated-access and efficient processing of health information.

This article is a step towards developing such a system. We will look at existing systems and identify their functions and structures. Special attention will be devoted to assessing the level to which the reviewed software systems serve the general requirements of HIS including: scope of content, information capture, report generation, information representation, decision support, security and confidentiality, performance, interoperability and adherence to software engineering principles.

The rest of the paper is organized as follows: section 2 is a general overview of HISs in Oman including geo-political and medical aspects; section 3 contains a review of common HIS functionalities and standards; section 4 provides a description of two exemplary and most commonly used systems in Oman; and section 5 is the conclusion.

\section{Health information systems in Oman}

The Sultanate of Oman has a wide geographical area with a sparse and small population scattered all over the country. This poses a great challenge to health care delivery. There is also movement of inhabitants between the country side and major cities. Some of its nomadic inhabitants (Chatty, 1983) also move around periodically with the change of seasons, adding more difficulties to the healthcare management.

Oman, a developing country, has a lot to gain from information technology in the field of health care. The topology of the country and the demography of its population lend themselves well to the use of enabling technologies in health care. HIS can expedite the delivery of the much needed health services and improve the utilization of existing services provided by the Ministry of Health and its affiliated institutions including in particular the Sultan Qaboos University Hospital (SQUH). Existing systems can benefit from some improvements in a number of areas mainly coverage and integration.

\subsection{Oman geo-political and medical perspectives}




\section{REVIEW OF HEALTH INFORMATION SYSTEMS IN OMAN}

Although Oman is an upper middle income country, offering healthcare to its small population is hampered physically and financially by its relatively large size and difficult terrain. Oman's population is scattered over large areas of sparsely populated settlements, many with fewer than 500 inhabitants. Expenditure on healthcare has, however, made quantum leaps. As of 2004, the results are impressive: 56 government hospitals, 166 government health centers, clinics and dispensaries, 13.9 doctors and 35.2 nurses and midwives per 10,000 inhabitants. Life expectancy today stands at an average of almost 74 years compared to 49 in 1970, and infant mortality per 1,000 is down from 118 to 16.2 by 2004 (ESCWA, 2005) (EMRO, 2006).

Oman's healthcare system today is a public model, with health services predominantly financed and provided by the government which accounts for $95 \%$ of all hospitals, $80 \%$ of doctors, $94 \%$ of nurses and $83 \%$ of other paramedic professionals. Although there are 631 private clinics, just $20 \%$ of these provide limited specialty care (Wilkie, 2005).

The present responsibilities of the Ministry of Health (MOH) as stated in the Sultanate of Oman National Health Policy Statement (Ministry of Health , 1992) include:

- Establishing the health policies in the general frames of the country's strategy.

- Planning the health care human resources in the framework of the country's strategy and this includes establishing health institutes in different specialities.

- Providing information and health statistics and conducting studies in the different health areas.

- Providing health services (preventive and curative) for the citizens and residents of Oman.

- Conducting training and skill development programmes for the medical staff.

- Co-operating with medical colleges in training of medical students in the MOH institutions.

- Educating people about health issues and encouraging them to take care of the hygiene and of their health.

- Working on improving the coordination with Gulf Cooperation Council (GCC), Arab countries and international community in health matters.

Oman is divided into 11 health regions. Each health region is autonomously operated and headed by a Director General of Health Services reporting to the Undersecretary of Health Affairs. The health policies and guidelines are formulated by the Directorate General of Health Affairs. The health service in Oman is divided into Primary, Secondary and Tertiary care services. The Primary Healthcare is mainly ambulatory service except in some regional health centers where they have delivery facilities. There are also some Wilayat (States) hospitals or local hospitals which deliver only Primary Healthcare services.

The Secondary Healthcare comprises Polyclinic or Extended Health Centers, which are ambulatory and Referral Regional Hospitals with Inpatients facilities. There are four main Tertiary Healthcare hospitals, all located in Muscat and each of which is dedicated to certain specialties. Those hospitals are the Royal Hospital, Khoula Hospital, Nahdha Hospital and Ibn Sina Hospital. All hospitals report to the Undersecretary of Health Affairs except for the Royal Hospital which reports directly to the Minister of Health.

The Referral Hospitals and the Tertiary Hospitals in Muscat are autonomous in their operations and managed by an Executive Director, with the exception of the Royal Hospital which is managed by a Director General.

The Ministry of Health is looking at further streamlining of its operations. The ministry has been running a pilot study experiment in Muscat governate for the last three years with the intention of extending the autonomy scheme to the Wilayats.

\subsection{Health plans and e-government initiative}

The Omani government has made it a strategic goal to use information technology as a tool in supporting its operation and delivery of services with the e-Government initiative. The National IT Authority was created to streamline and coordinate all the IT activities by creating national- and sector-wide group of interest. Several projects such as the Government Network, a high bandwidth Multi-protocol Label Switching (MPLS) network, and the Educational Portal have already been initiated. As part of the e-Government initiative, the Ministry of Health would have to propose a nation-wide e-Health Portal Plan that would link together all the healthcare 


\section{MOHAMMED ELHADI, ET AL}

providers in order to exchange the necessary health information and also to build up the National Unique Patient Repository.

This is in-line with international aspirations and with the World Health Organization (WHO) Resolution (WHO, 2004), passed by the $58^{\text {th }}$ World Health Assembly, calling all the member states to have a National eHealth Strategic Plan that would link together all the institutions providing healthcare services.

\subsection{Oman health service providers}

A number of health care providers exist in Oman ranging from $\mathrm{MOH}$ to some private providers. The majority of the services, however, are provided by $\mathrm{MOH}$ which provides about $90 \%$ of the health care in Oman. Other important players include Sultan Qaboos University Hospital (SQUH), Armed Forces hospitals, Royal Oman Police hospitals and Petroleum Development of Oman (PDO) hospitals.

\subsubsection{Ministry of health}

The initiative of using information technology to support healthcare activities has started since the commissioning of the Royal Hospital in 1986 to buy a system running only on WANG computers. The system turned out to be expensive to acquire and maintain and did not fulfill the requirements of the hospital and the Ministry of Health. This failure has hampered the utilization of IT in healthcare in the whole Ministry of health for a very long time.

In the early 90's, the Ministry of Health decided to look seriously at the wide utilization of IT in the healthcare system and all the other support to the healthcare activities in the Ministry. A specialized dedicated unit to look at IT activities in the whole Ministry of Health was created. A team comprising, clinicians, other medical staff, administrators and IT staff was commissioned by the Minster of Health to advice him on the utilization of information technology tools in the healthcare institutions.

The main recommendation of the team was to acquire internal expertise for developing in-house all the software requirements to manage the healthcare activities. In 1997, the first pilot project at a health center, Wadi Al Jawahir in the Suwaiq Wilayat was successfully completed. In 1998, Nizwa Hospital was equipped with a full fledged HIS. Since then a massive deployment program has been initiated. There are over 140 fully computerized health institutions to an almost paperless status and 3 hospitals are film-less.

The strategy adopted by the Ministry is to make computerization an integral part of new institutions. Every year some selected old institutions are included in the computerization program based on the size and the workload. All major institutions are already computerized including the National Referral hospitals, the Regional Referral hospitals, the polyclinics, extended health centers, and the health centers built after 1995 .

\subsubsection{Sultan Qaboos University Hospital}

Sultan Qaboos University Hospital (SQUH) has started its operations with a health information system package called Gerber Ally, which was selected from a tendering process. Realizing the need for a better system, SQUH floated a tender for a new HIS package. After lengthy evaluation, TrakCare HIS package was selected and it is currently in use.

\subsubsection{Other health providers}

A number of other providers do exist in Oman but are mostly independent institutions. They include:

- The Army Medical Services (AMS). They purchased an HIS package from a local supplier. The acquired package did not cover all the processes in the hospital. Processes such as the laboratory, pharmacy, and clinical documentation were still manually performed. At the beginning of 2006, the AMS acquired a new health information system which provides the necessary functionalities of the hospital.

- The Royal Police Hospital started with a pilot project that provided an archiving system (document scanning). However, the implementation was not very successful, and at the beginning of 2000, a new Hospital Information System was acquired. 


\section{REVIEW OF HEALTH INFORMATION SYSTEMS IN OMAN}

- $\quad$ PDO has also acquired an HIS package, which is running for several years now. The maintenance and support of the package are still a concern for PDO.

For the private health institutions, automation of health care still needs to be put as a high priority. Patients' billing is the main concern at this time. It is the only fully computerized process.

\section{HIS requirements, functionalities and standards}

Importance of HISs requires their professional development and conformance with high standards. Any HIS has to adhere to standards and provide for some common and basic set of accepted requirements and functionalities.

Many standards have been emerging but there still is no fully developed and accepted international single standard. As such, we have decided to approach the issue from two perspectives. On one hand we looked at commonly desired features and attributes and on the other we surveyed available standards. Commonly desired features are in a way, a bottom up approach that would list essential and desirable features current systems have or should have. We are adopting a list of functionalities surveyed in literature and expressed by some of the constituents we have been talking to in the course of the survey. In particular we have heavily followed and used the functional categorizations provided by Beale (2001) which divides the features and functionalities of HIS systems in the following manner (each of which is further elaborated on in section 3.2):

- Minimal functionalities: minimum set of services to be provided by any HIS.

- Full functionalities: a set of functionalities that are optionally provided by HISs.

- Enterprise Functionalities: A much wider and more complex set of functionalities that can be provided by systems to support enterprise-wide use.

- Local Requirements: A set of functionalities derived from local and regional geo-political, legal and medical nature of the country and the region. This set relates to data ownership, legal procedures for security and confidentiality and similar issues of concern in Oman.

\subsection{The electronic health record}

An important and focal component of any health systems is the health record and of particular interest in any HIS is the concept of Electronic Health Record (EHR).

\subsubsection{Definition}

There is no single agreed upon definition for the electronic health record or EHR. It has been called differently at different times and places including: Computer-based Patient Record (CPR), Electronic Patient Record (EPR), Electronic Health Care Record (EHCR) and National Health Record (NHR).

Many definitions are cited in the literature (NHR Task Force, 2002) (Handler et al, 2003). EHR is a repository of patient-centered records containing contributions by health care providers in the course of a lifelong care process. Centered on patient's information, the EHR automates and streamlines the clinical workflow taking care of communication loops that result in delays or gaps in care.

EHR supports data collection and storage for different types of users ranging from clinical to administrative including billing, quality assurance, reporting, planning, monitoring and reporting.

\subsubsection{EHR content, usage and ownership}

A number of issues are the subject of debate in the literature on what makes up EHR, their usage and ownership. Many models were proposed that describe the content of an EHR (Sowa, 2000, Bird et al., 2003). The basic data content of EHR includes but not limited to: 


\section{MOHAMMED ELHADI, ET AL}

- Persistent information: this is typically static data that remains valid for the life of the record. It includes patient identification, medical, family and social history, medications, therapies, precautions (such as allergies), drug intolerances, immunizations and vaccinations.

- Test results: records of data and results of tests undertaken by a patient.

- Healthcare professionals' input and contribution such as histories, examinations, diagnoses, orders, care plans and summaries.

Another type of data advocated by many researchers (Beale, 2001) to be included in EHR is "context" information. Each item of knowledge in the EHR relates to certain time, place, clinician and patient particularities during care. Numerous other data items that should be included in EHR record have been cited and discussed in the literature. (Beale, 2001) contain discussions of items such as orders and pathology, vital signs data such as "diagnostic sequences", imaging, and multimedia items, demographic information, and clinical guidelines. It should be noted, however, that not every aspect which concerns the subject of care is of interest to every EHR user.

Clinical data and knowledge content of the EHR is provided and used by different constituents. Sources of such knowledge include:

- Clinicians who provide their observations, summaries and care plans.

- Associated health workers such as social workers and nurses, who enter their observations and measurement.

- Laboratory staff that provide raw data such as test results.

- Patients: who provide symptoms, complaints, etc.

Healthcare professionals need to be able to use the data in the EHR to support views and high level concepts such as:

- Trends

- Timeline views and problem threads.

- Automatic processing, decision support and statistical and epidemiological systems.

The issue of ownership of EHR is unclear and is subject to the level of maturity of the society. It is influenced by:

- Ownership of the technical infrastructure on which the records reside.

- Responsibility for data management.

- Legal ownership of the content.

- Access rights for public, research, and business sectors.

Consensus appears to exist on the right of the consumer to the ownership of health record.

\subsubsection{Architecture and implementations}

As this paper does not concern itself with architectural and implementations issues of the EHR, we would just like to mention the following in this respect:

- Architecture undoubtedly matters as far as quality of HIS systems is concerned.

- Many proposed architectures and implementations are provided (Beale, 2002, Bird et al., 2003, HL7, 2004, Katrhakis and Tsiknakis, 2006).

\subsection{HIS important functionalities}

As have been mentioned in the previous sections, and with lack of an agreed upon standard, we adopted and closely followed the classifications used by Beale (2001).

\subsubsection{Minimal functional EHR environment}

In reality EHR is complicated, non-standardized and fragmented across many providers. Clinical care and administration routines can start with minimal functionalities. The following are some exemplary but limited services that an EHR should provide: 


\section{REVIEW OF HEALTH INFORMATION SYSTEMS IN OMAN}

\section{Identification service}

A minimal identification service containing sufficient demographic information on patients, health professionals, and institutions is needed. Light Directory Access Protocol (LDAP) service or Patient Identification Service (PIDS) are normally used to provide this functionality.

\section{Terminology}

Controlled vocabularies and terms from multiple vocabularies are typically made available by a single service in the HIS environment. Such services are used by decision support and by clinical models. An example of this is the Terminology Query Service (TQS), or Lexicon Query Service (LQS), of the Object Management Group (OMG). This enables heterogeneous systems to publish their medical terminologies in a form that can be queried for equivalences and definitions independent of the underlying implementations. Digital Imaging and Communications in Medicine (DICOM) is a standard for handling, storing, printing, and transmitting information in medical imaging. It includes a file format definition and a network communications protocol. The National Electrical Manufacturers Association (NEMA) holds the copyright to this standard. International Classification of Diseases (ICD) is used to standardize classification of diagnoses, procedures and pathological classification.

\section{Reference data}

Good level of reference data for drug descriptions is essential.

\section{Clinical models}

Health information systems based on formal domain models are known as "archetypes". These are models used to define rules for specific clinical concepts used in the health records. They enable significant improvements in both the clinical quality of systems, as well as their economics. The Good European Health Record and SynEx systems both include archetype repositories.

\section{Query and update service}

Primary interface to the EHR to be used by applications and users is an integral part of EHR.

\subsubsection{Full functional environment}

A more capable and complete HIS would include some or all of the following:

\section{Workflow management}

Care delivered by multiple parties is represented as a network of actions by professionals or providers. Rather than being managed on ad-hoc basis by medical staff, it would be more efficient and complete if it can be managed as a process using automated workflow systems.

\section{Event management}

Events and orders make up a big part of the actions that take place in a health system. A record of the major actions, such as prescriptions, requests for pathology and surgery are included in the HER but many of the many intermediate steps (e.g. the more complex pathology tests, surgery) are not of interest in the HER. Such events are essential for order management. Many orders take place in the context of well-known workflows.

\section{Multimedia and genetics}

Imaging test results such as x-ray, ultrasound, Magnetic Resonance Imaging (MRI), nuclear scans, electrophysiology such as Electrocardiography (ECG) and Electroencephalogram (EEG), and genetics data make up the most detailed and bulky data. Such data are logically integrated with the EHR, but usually served from 


\section{MOHAMMED ELHADI, ET AL}

specialized computer systems with special storage devices designed to satisfy the storage and retrieval patterns associated with large data. Specific items, usually of diagnostic significance, may be added to the EHR.

\section{Demographics and party identification}

Most providers record more demographic information than needed by the simple ID service in a basic environment. Such information is usually for practice management, billing and administration, but may also be clinically relevant. Most health bodies have a "minimum data set" which includes a large number of demographic details for patients and health professionals. PIDS is possibly the most appropriate service for demographic information and identification in health environments today.

\section{Guidelines, protocols and care pathways}

Guidelines and protocols are considered as the decision-making and planning tools of the clinician. Their use allows other users to understand the reasons for decisions, so that investigations can be effective. Work in the guidelines area includes the Arden Syntax (Pryor et al, 1993) (Arden Syntax), the GLIF language (GLIF), Asbru (ASBRU), and ProForma (ProForma).

\section{Decision support}

Decision-support systems require disciplined patient data organization in order to function properly. They rely heavily on coded terms, and formal clinical models in order to navigate data intelligently. In secondary care contexts of the future, decision support may be the primary reader of EHR data.

\section{Clinical modeling}

Clinical models are representations of physiological and pathological phenomena that are used for predicting patient evolution. They may be locally developed in more advanced provider institutions, typically those attached to universities or medical colleges. Such organizations are likely to be a source for clinical models for other providers to use.

\section{Mobile computing}

Hand-held computing devices are becoming more common in hospitals and health care institutions. The EHR needs to be available for viewing and potentially updating via relatively low-bandwidth interfaces, which may also need to support asynchronous transfers.

\subsubsection{Provider enterprise functionalities}

Enterprise-wide care is the ultimate objective of health care. It requires that HIS have the following capabilities:

\section{Administration and billing}

Managing beds, operating rooms and other resources along with the ability to communicate relevant financial documents between patients, other providers and health insurers or the government is an important consideration.

\section{Resource location}

This is an enterprise-level service that responds to distributed queries in the open environment. It enables requestors to know what information is available inside the provider environment.

\section{Portal}

An enterprise-level access provided to satisfy the various needs of not just clinical users, but also of population queries, government, insurance organizations, and patients themselves. 


\section{REVIEW OF HEALTH INFORMATION SYSTEMS IN OMAN}

A number of prototypes have been built indicating likely directions where patients would be able to control access to their records, via an "eConsent" mechanism. They would be able to enter their own data and to perform basic queries and obtain summaries of their health records.

\section{Data sources - investigations}

Raw data comes to the EHR from numerous sources including provider institutions and home such as wearable devices. Data used is likely to be formatted using a messaging standard such as HL7 or EDIFACT to guarantee some level of uniformity for the information receiver.

\section{Outside the provider enterprise}

Parties outside the provider enterprise such as patients, government agencies, insurance organizations, researchers and educators interface with the services provided inside the enterprise via a secure gateway, typically implemented as a web portal interface.

A patient's health record information may reside in more than one provider environment, and is logically assembled via distributed web clients which make use of the resource location facility to determine where data exists for a given patient.

\section{Online resources}

Numerous online resources which are used and operated by EHR environment providers are beginning to appear including terminologies, clinical guidelines, drug and interaction data, public demographics, and libraries of domain models. Making use of such resources would be an important cost-effective measure.

\subsubsection{Other functionalities and requirements}

Another set of requirements that we consider important are listed in this section. Some of these requirements are local to Oman and are based on local geopolitical, legal and medical issues (such as data ownership, legal procedures for security and confidentiality).

\section{Confidentiality legislation}

Oman like everywhere else needs to develop proper legislation to regulate patient data confidentiality and access. Patient information should be held under legal and ethical obligations of confidentiality. Information provided in confidence should not be used or disclosed in a form that might identify a patient without his or her consent except in circumstances where it is reasonable to expect that the information will be held in confidence. Patients trust the providers or allow them to gather sensitive information relating to their health and other matters as part of their seeking treatment. They do so in confidence and they have the legitimate expectation that staff will respect this trust.

\section{Ownership regulations}

Data contained within the medical record belongs to the patient, whereas the physical form which the data takes belongs to the entity responsible for maintaining the record. In many countries the patient has the right to ensure that the information contained in their record is accurate. Patients can request their health care provider to remedy factually incorrect information in their records. 


\section{MOHAMMED ELHADI, ET AL}

The use of a unique national Medical Record Number (MRN) is a critical issue for any HIS. It provides accurate and efficient access to the patient medical information whenever and wherever it is needed. In Oman, this issue is currently being addressed and investigated and work is in progress to come up with a solution.

\section{Common repository}

Data storage is another important factor in HIS. More than one approach exists. A choice has to be made whether to use a national common repository for all health information systems or to have data distributed among providers in Oman.

\section{Integration with existing systems}

Due to the existence of different systems in the country under public, private and other domains, systems will need to be integrable and interoperable.

\section{Support for educational and research work}

Need arises in many hospitals and medical institutions for data and systems to be available for educational, training and research purposes. HIS will need to be able to support such needs technically and at the same time address the confidentiality and security of the information being used.

Beside the educational module, the system can support also the researchers by allowing the developer to write reports to extract data required for the researchers. For example the system should allow searching for a specific disease using different criterion such as time span, gender and similar restrictions. The system should have online facility to provide some statistics enquiry for specific information that can help the researches in analyzing and finding the right information from the system.

\subsection{EHR standards}

The main purpose for developing and adopting national and international EHR standards is to facilitate the achievement and improvement of the EHR functionalities and requirements. Interoperability is one of the key EHR requirements. It is the most lacking for information management and will greatly benefit from EHR standardizations (HL7, 2004, Katrhakis and Tsiknakis, 2006).

The three main organizations currently active in EHR standardization are ISO (International Standard Organization), CEN (Committee European Normalization - the European Standard Organization), HL7 (Health Level Seven) (HL7, 2004, Katrhakis and Tsiknakis, 2006, Bott, 2004, Schloeffel, 2004). The openEHR foundation (openEHR) and Integrating the Healthcare Enterprise (IHE) are not health standard bodies but they have made an important impact on the EHR standardization. The following subsections provide an overview of the five organizations and their roles in EHR standards.

\subsubsection{ISO / TC 215}

The (ISO) Health Informatics Committee (ISO/TC 215) is the main international organization for health information standards. It currently has 25 participating countries and 16 observering countries. Some of IOS/TC 215 EHR standards are completely developed by TC 215 working groups, while others are developed using existing EHR standards from other national and international organizations such as IEEE, CEN, and HL7 (Ocean Informatics, 2007, Schloeffel, 2004).

TC 215 is currently composed of 9 working groups each focusing on important issues such as data structure and interchange, security devices, ... etc (ISO/TC 215).

\subsubsection{CEN/TC 251}




\section{REVIEW OF HEALTH INFORMATION SYSTEMS IN OMAN}

The Committee European de Normalization (CEN) is the main organization for standards for those countries that are members in the European Union. CEN/TC 251 is the health informatics technical committee of CEN. It is composed of the following four working groups (CEN/TC 251):

- WG1: Information Models - Developing EHR models and general message descriptions.

- WG2: Terminology and Knowledge Bases - Semantically organizing health information so it can be used in the domains of healthcare informatics.

- WG3: Security, Safety and Quality - Developing techniques for protecting confidentiality, integrity, availability, accountability, and security.

- WG4: Technology for Interoperability - Working on middleware, medical imaging, multimedia, and medical device communication in integrated healthcare.

CEN/TC 251 has two EHR standards which are developed by performing major revisions for its previously published pre-standards (HL7, 2004, Schloeffel, 2004). The first standard is CEN 13606 "Electronic Health Record Communication”. It consists of 5 parts: (1) Reference Model, (2) Archetype Interchange Specification, (3) Reference Archetypes and Term Lists, (4) Security Features, and (5) Exchange Model.

The second standard is CEN 12967 "Health Informatics Service Architecture" (HISA) which is a high-level service-based architecture. HISA standard is composed of three parts: (1) Enterprise View, (2) Information View, and (3) Computational View. It provides a reference model for health care information technology services, thus facilitating building and purchasing of interoperable EHR systems.

\subsubsection{HL7}

Health Level Seven (HL7) is US-based, not-for-profit, ANSI-accredited health information standard organization (Ocean Informatics, 2007, Shaver, 2006) (HL7). It has more than 2,400 members including 90\% of the largest healthcare information systems venders. HL7 is traditionally used to develop specifications for application-level messaging between health information systems (i.e., interoperability standards). In 2000, its mission was revised to support the electronic health record (EHR).

HL7 "Version 2.x" messages standards are widely used in the United States and other countries. These standards are typically used between health information systems inside the same hospital, and/or between hospitals and external laboratories.

In 1997, HL7 version 3 (or v3) was developed with a new set of standards. Its aim was to define application messages based on formal models. A restricted process is used to construct message content schemas including the reference information model (RIM), domain information models (DIM), restricted message information models (RMIM), common message element types (CMET), and hierarchical message definition (HMD). HL7 version 3 uses Extensible Mark-up Language (XML) to generate message schemas.

The Clinical Document Architecture (CDA) is the first EHR-related HL7 standard (HL7, 2004). CDA is an XML specification for clinical documents. It forms an important sub-component of the EHR (not full EHR specifications) and is compatible with the equivalent components in CEN 13606 and openEHR standards.

\subsubsection{Open EHR}

The openEHR foundation is an independent, not-for-profit organization that brings together an international community to facilitate creating and sharing of electronic health records via open-source, standards-based implementations (Beale and Heard, 2006). It was jointly founded in 1999 by the University College of London and Ocean Informatics.

OpenEHR uses a two-level modeling approach to define a common specification for sharing electronic health records (EHRs) within an open architecture (Garde et al, 2007). The two-level modeling is used to separate knowledge from information. The first level is the reference information model which can safely store and exchange any clinical information. The second level is used to model the knowledge using openEHR archetypes.

OpenEHR formalizes the electronic health records in terms of Reference Model (RM), Archetype Model (AM), Service Model (SM), and Terminology and other ontologies (openEHR). OpenEHR uses an XHL-based data 


\section{MOHAMMED ELHADI, ET AL}

format to support the sharing of EHR data. It uses a consistent approach for transmission and storage of EHR. It is compatible with other EHR standards such as HL7 version2.x messages and HL7 Clinical Document Architecture (CDA).

\subsubsection{Integrating the healthcare enterprise (IHE)}

Integrating the Healthcare Enterprise (IHE) is not-for-profit multinational healthcare initiative aimed to accelerate the adoption of information standards needed to support EHRs. IHE was jointly founded in 1998 by the Healthcare Information and Management Systems Society (HIMSS) and the Radiological Society of North America (RSNA).

IHE does not develop new EHR standards but it rather drives the adoption of EHR standards to address specific clinical needs (Eichelberg et al, 2005) (IHE). IHE Integration profiles are used to select and recommend appropriate EHR standards for a specific healthcare need, develop restrictions, eliminate ambiguities, reduce configuration and interface costs, and ensure high levels of interoperability.

IHE has developed a unique process for EHR standards adoption (IHE). The process involves repeating the following major steps in a yearly cycle:

- Identifying interoperability problem by clinicians and IT experts.

- Integrating Profile Specification. Health care IT professionals identify relevant EHR standards and specify how to apply them to address the specified problems.

- Testing systems at the IHE Connectathon. Vendors implement IHE integration profiles for their products, and test their products for interoperability at the annual IHE Connectathon.

- Publishing Integration Statements. Two steps are performed to simplify the systems acquisition process: (a) venders publish IHE integration statements for their products, and, (b) users can refer to the IHE integration profiles in requests for proposal.

\subsubsection{Harmonization}

The harmonization of EHR standards is extremely important to facilitate the interoperability between different health information systems that use different EHR standards. IOS/TC 215 has performed a very important role in EHR harmonization at the international level (HL7, 2004). The three main standard development organizations (ISO/TC 215, CEN/TC 251 and HL7) have signed agreements and MoUs (memorandum of understanding) to further coordination and collaboration among them with particular emphasis in EHR harmonization (HL7, 2004, Schloeffel, 2004, Ribick, 2006). They have developed shared plans to strengthen the delivery of standards-based health solutions for all customers, and improve the safety, accessibility, quality, and effectiveness of health service delivery.

\section{Main HIS systems in Oman}

The Ministry of Health $(\mathrm{MOH})$ is the main provider of health care in Oman. MOH has a comprehensive HIS system called Al-Shifa (Arabic for recovery). It is an automation of most processes of healthcare delivery in $\mathrm{MOH}$ hospitals and health centers. The second most important HIS system in the country is the Sultan Qaboos University HIS system called TrakCare. Given the importance of these two systems and their coverage of an estimated $95 \%$ of HIS usage in the country, we present in this section a detailed description of these two systems.

There are over 140 computerized health institutions in Oman. All major institutions are already computerized. In addition, the strategy states that the usage of IT in all processes of the heath cares delivery system in order to streamline them and make them cost effective. It also calls for making of all IT applications tailored to all requirements of the local health institutions, and also providing necessary information for planning and other research purposes.

\subsection{The MOH Al-Shifa system}




\section{REVIEW OF HEALTH INFORMATION SYSTEMS IN OMAN}

The computerization in MOH started in 1987, at the Royal Hospital, a national referral hospital. In 1990, a specialized and dedicated unit for IT was created in the Ministry. In 1997, the first computerized health center was implemented using an in-house developed system. In 2004, the Information Technology unit of the $\mathrm{MOH}$ was upgraded to the level of Directorate General with 4 departments and 15 sections, to become the Directorate General of Information Technology (DGIT). The e-Health strategy of the Ministry of Health is built around Al Shifa. Each healthcare institution has Al Shifa customized to fit its specific requirements.

As a second phase, an e-Referral Engine has been built to allow the exchange of necessary medical information. It covers the request for appointment for further specialized treatment, investigation referrals, request for second opinion and referrals feedback. The system has also a management module, which provides the status and the necessary management information for referrals. The system is under implementation. $\mathrm{MOH}$ expects to complete the implementation of all healthcare institutions linked to the Ministry head-quarters by the end of 2007. Other institutions outside $\mathrm{MOH}$ would have to use it for transferring patients to the Ministry of Health institutions after 2007. The e-Referral Engine has been built on the guidelines for referral established by the Directorate General of Health Affairs

The third phase would be an establishment of unique identification and building a national e-health record repository. For the unique identification, under the e-Government initiative, the existing National Identification Number for Omani and the Residency Number for expatriate have been selected.

The Ministry of Health is exploring the feasibility of establishing the National e-Health Record Repository with the necessary securely mechanisms.

\subsection{System overview}

Al-Shifa Hospital Information Management System is a Client-Server system. It is a fully integrated graphical user interface application system for hospital management that is built around Oracle 9i database and developed using Oracle Developer 6i that runs under the platform of Windows 2000/Linux. The system uses a single menu that represents the fundamental components of the electronic medical record. It allows on-line access to admission, discharge, transfer, radiology and laboratory test results, etc. as per user access privileges.

\subsubsection{Main features}

The system was built to fit the local environment and it complies with user requirements supporting easy electronic exchange of medical information among health institutions. Adherence to international standards and interoperability were considered during the design and implementation phases.

The whole system (including the source code) is owned by the MOH. Therefore, any modification or customization is done in a cost effective manner. The system maintains an audit trail that records the previous information prior to making the changes, the changes made, the user's name that made the changes and the date and time of the changes. The system has been built in compliance with e-Health's internationally recognized standards, namely the HL-7, the CEN/TC-251, the ISO-215, SNOMED-CT, etc. Short messeging (SMS) and email services have been integrated in the system and are used to inform and remind patients about thier appointments in the various health care institutions. The system provides comprehensive data inquiry reports based on the user-defined search criteria.

\subsubsection{System description and functionality}

Al-Shifa Hospital Information Management System is made of the following components:

\section{Patient and clinical information management}

The Clinical System is a sub-system of the HIS that brings a patient's comprehensive medical record to a single screen. It provides the physician instant access to almost any desired level of underlying clinical details. The system also protects information from inappropriate access and provides control over who is exactly permitted to read, write and delete patient information. 


\section{MOHAMMED ELHADI, ET AL}

The Clinical System is represented by sub-modules and processes which allows doctors to perform tasks such as visit creation and admission, patient's laboratory and radiology orders, examination and medication, diagnosis and progress, vital information and history, nursing notes and other information relating to a patient's care.

The Clinical workbench enables users to take notes, place new orders for the patient, review existing orders, pre-operation orders, inpatient discharge orders, etc. Doctors can access the notes and records entered by the nurses such as vital signs and nursing notes, Intravenous (IV) fluids, nursing procedures, medication executions and other related nursing care.

\section{Medication and pharmacy management}

The Medication module automates the process of writing and tracking prescribed medications. All medications are recorded regardless of the patient's visit status whether the patient is Outpatient Department (OPD), Accident and Emergency (A\&E), inpatient or daycare.

The medication module is interfaced with the Pharmacy module so that prescribed medications are automatically sent online for quick dispensing. A facility to view the non-availability of the drug in the pharmacy is provided to inform the doctor of the availability of particular medicine.

The Medication module is also interfaced with the medication dispensing in the Nursing module so that prescribed medications for inpatients are carried out according to the dosage and course prescribed by the doctor. Prescribed medicine deletion is allowed only if the medicine has not been dispensed to the patient. If it is decided to discontinue the ongoing medication, the system records the date and time and the name of the doctor stopping the medicine.

The Medication History module maintains the details of each medicine being prescribed. It includes the order date, the dosage details, the doctor's name, stop date and the reason for discontinuing. It also maintains a record of dispensing details such as dispensing quantity, dispensed location and the name of the staff that administered the medicine. Medication literature and overview are available and could be seen by the doctor prior to medicine prescription. Information included in the literature relates to dosing information, pharmacokinetics, cautions and clinical applications.

\section{Radiology and clinical laboratory management}

Requesting laboratory tests is a quick and easy process, which uses a single screen to enter the test information. A wide variety of ordering parameters allows the doctor to designate test orders and set priorities. The system has also the ability to alert the doctor if a repeated order is made as well as the cost of each investigation.

The Visit History function provides a complete record of the previous laboratory investigation details arranged according to the type of visits. Within each visit, the date, time and the details of the results of each investigation are recorded.

The Blood Request function is interfaced with the laboratory system that enables the doctor to order online blood bags required for a patient's surgery procedures or in any given situation wherein a blood transfusion is required. Blood requirement details such as blood type, blood group, bag and required volume, required date and the reason must be filled in.

The Ultrasound module runs as a part of the clinical system allowing the doctor to enter the examination results of the fetus and the female genital tract.

The Laboratory system has the facility to maintain an audit trail that records the previous investigation results prior to recall.

\subsubsection{System's protection and security}

The system has been designed with compliance of the main elements of e-security: confidentiality, integrity, and availability. The system is isolated physically from the Internet. It does not allow the use of removable storage such as USB memory and CDs within the Health Net. 


\section{REVIEW OF HEALTH INFORMATION SYSTEMS IN OMAN}

Within the system, access to the different functions and processes are controlled through strong users' profiles and by user identification and password to restrict unauthorized users from gaining access to other modules from which they are prevented from entering or updating. The critical components are implemented in redundant fashion to insure high availability to the patient record.

The e-security team in DGIT is working on implementing an e-security policy, which includes many instructions to the system users and administrators. The system provides full data security that can limit specific functions and process by username and password.

In addition, the system provides control over access to menus, icons that represent a particular process and functions for each user or groups of users.

The system can identify the user of a particular function or process so that an audit trail of changes made to the patient record is maintained. The system records the previous data, the changes made, the user who made the changes and the date and time.

\subsubsection{Future plans}

The following are $\mathrm{MOH}$ planned future extensions to Al-Shifa system:

- A national Repository of $e$-Health Record

- $\quad e$-Referral Engine, which automates the following functions:

- Request for appointment

- Consultation feedback

- Request for second opinion

- Investigation referral

- Creating e-Health Legislation

- Links among all health institutions

- Use of national ID number

- Tele-Education Project

- Integration of Financial, HR, Administration and Clinical Systems

- Disaster Recovery System

\subsubsection{Challenges}

The following are difficulties to related to the adoption of the MOH Al-Shifa system:

- Lack of IT experienced technical staff

- Lack of training programs for medical staff.

- No standard requirements (each hospital has its own requirements)

- Cost of e-links (expensive)

\subsubsection{Levels of functionality and compliance with standards}

AL-Shifa system provides for the most basic functionalities any HIS system is expected to do, taking relevant standards into account. However, it lacks formal commitments to standards. It is expected that once the systems is fully functional and all planned enhancements are done, it will comply with major international standards.

\subsection{The SQU trakcare system}

The Sultan Qaboos University hospital uses an HIS system called TrakCare. It is an integrated hospital management system that provides computerized support for a number of clinical, administrative and financial operations at the hospital. The SQU Hospital started using this new TrakCare system in July 2006, replacing an older HIS System which was in use since 1992. TrakCare is a ready made package System developed in Australia. This section describes to some level of detail the TrakCare system. 


\section{MOHAMMED ELHADI, ET AL}

\subsubsection{System overview}

TrakCare is an integrated hospital management system composed of a set of components serving the different needs of the various hospital departments as discussed in the next section. The system uses a proprietary relational database management system called Cache. At the heart of the system is the Patient Administration and Clinical System (CIS, PACS, DICOM, File Systems) along with the Integrated Human Resource and Finance Management System (HRFMS) Application. Remote access to the System can be provided using the internet.

\subsubsection{Main features}

One of the main features of TrakCare is the ability to define patient and data flows. The system can be configured to route information relating to patients to specific locations or departments in the hospital based on the defined hospital policies. In addition, the system allows users to define clinical pathways and use them to route patients. TrakCare is a web-based system which allows remote access using Internet connections. The system also provides mobile support allowing users to record information at the point of care. It is possible to link to any other system or devices conforming to the HL7 protocol. The system provides access to live data for the medical students to work on without affecting the original data which is a good feature for the University Hospital.

\subsubsection{System description and functionality}

TrakCare is composed of different modules serving the needs of the various hospital departments. The following are brief descriptions of the main system modules:

\section{Patient administration system (PAS)}

The patient administration system is the entry point of patients to the medical facility. It provides functions to manage patients from the point of admission until discharge. It involves patient registration, admission, inpatient and outpatient management, billing and medical records.

\section{Clinical information system (CIS)}

The clinical information system provides the necessary tools and functions to manage patient clinical data. The clinical information system is further divided into sub-modules which allow the management of clinical records, patient orders, medical and nursing notes:

- Electronic Patient Record: Alerts, Allergies, Patient Medical History, Family-History and Interactive Icons.

- Operation Notes: Pre-Anaesthesia, Post Anaesthesia and OT Notes.

- $\quad$ Student Electronic Patient Record (SEPR)

\section{Pharmacy management system (PMS)}

The pharmacy management system supports the workflow of inpatient and outpatient pharmacies. PMS provides formulary, dispensing, and manufacturing functions. It supports the pharmacy's day to day work cycle from ordering till dispensing. Also this module links with inpatient medicine dispensing and substitutions medicine.

\section{Radiology management system (RMS)}

Currently SQUH is using RMS from Siemens which is going to be interfaced with TrakCare for the necessary data, Reports, Orders and images to flow between the two Systems. The RMS module provides functions to run and manage the radiology department. It provides scheduling, inventory, reporting, and film tracking. Results entry can be done using text or voice recording. 


\section{REVIEW OF HEALTH INFORMATION SYSTEMS IN OMAN}

\section{Laboratory management system (LMS)}

The LMS module provides support for the various laboratory departments. The system supports both uniand by- directional integration to most of the lab instruments. LMS provides the necessary functions to manage specimen receipts, results entry, and results authorization.

\section{Non-medical services}

TrakCare includes a number of non-medical support services for handling patient accounting, inventory management, and central supplies management.

Patient accounting: TrakCare includes a patient-accounting sub-system supporting the following:

- Accounting for individual patients.

- Accounting for corporate patients with and without shared costs.

- Insurance schemes with and without shared costs.

- Multiple payers per patient episode.

- Multiple insurance schemes.

The user can define the patient billing rules using a table-driven interface. Patient accounting reports can be generated regularly or on-demand. The patient accounting module supports financial management of multiple sites with autonomous or centralized control.

In terms of insurance management, the accounting module supports the handling of multiple insurance plans with user-defined parameters for each insurance plan.

A contract management feature supports the billing and collection of vendor billing accounts. Activities affecting a patient's account are recorded and transaction histories can be viewed on-line.

Inventory Management: This module allows computerized handling of purchasing and stock management. Purchase orders entry, approvals, and status tracking are supported. The purchase orders can be triggered by the inventory levels.

Individual stock items can be assigned batch controls, shelf life restrictions, and stock locations, replenish source, minimum/maximum quantity, re-order level and quantity.

This stock system of TrackCare is interfaced with the human resources and financial system to transfer data from main stores to various locations.

Supplies: This module allows management of sterile supplies, linen and disposable supplies. Supported functions include items categorization, sub-stores identification, and item-location association.

Additional Features: TrackCare includes a number of additional features supported for mobile as well as webbased access to medical information. The following features are supported by the system:

(1) Mobile Information System: Palm devices can be used for uploading, accessing and updating offline medical information during ward rounds and during home nursing visits.

(2) Web Interface: WebTrack supports web-based access using standard web browsers. The main supported web-based operations are patient registration, online appointments, access to patient records, and online orders.

(3) SMS: The Short Messaging System (SMS) for patient's appointment.

\subsubsection{Areas of improvement}

The TrakCare system supports many of the important health care functions as described above. Some areas that are missing or require improvement are listed below:

\section{Medical audit}

Medical audit is the evaluation of medical care in retrospect through analysis of medical records. It relates to examination and verification of dealings of medical care. Medical Audit is vital for the measurement of the quality of care given to the practice population. Medical audit requires standard setting, data collection, comparison with standards, review of data and standards, review of current practice, change in that practice and further data collection and comparison with the original data. 


\section{MOHAMMED ELHADI, ET AL}

\section{Medical referrals}

In order to obtain appropriate medical care, patients can be referred or transported from one hospital to another based on the capacity, capability and quality of medical care provided by hospitals. Therefore, enabling patient care records to be shared among hospitals is essential not only in delivering the quality of medical care services but also in saving medical expenses. There is a need for a unified referral information system in which patient care records can be shared among hospitals over the Internet.

\section{Mobile computing}

The ubiquitous access to information anytime, anywhere in the medical field is very important. One of the promising ubiquitous computing models is the mobile clinical information system using hand-held computing devices. The system should enable doctors to access patient clinical test results from any location, make clinical orders instantly, and send the consultation requests to the specialists using a portable device (Fischer et al., 2003, Ebell et al., 1997, Greiver, 2001).

\subsubsection{Integration with other systems}

With recent movements toward shared clinical data, integration between different medical information systems is becoming more and more important. Data integration, particularly in a medical environment, is an important issue that must be considered seriously. The integration of data from a variety of sources will improve the clinical decision-making process (Stead et al., 2000). Medical data representation and communication standards should be deployed for interoperability among various systems.

\subsubsection{Challenges}

Being an international system and the system is to be customized; the involvement of users was required in defining all aspects of the system.

The package lacked certain functionalities which are being built. The functionalities missing were IV processing, Discharge summary and Pharmacy processing for Total Parentental Nutrition (TPN), Diet processing, etc. Alternate manual procedures had to be found for these. Many of the users had very little experience in using computers for day to day work and hence took time to get used to the new system.

\subsubsection{Levels of functionality and compliance with standards}

The TrakCare system is compliant with the following standards:

- International classification of diseases (ICD)

- Digital Imaging and Communications in Medicine (DICOM)

- $\quad$ Systematized Nomenclature of Human Medicine (SNOMED)

- $\quad$ Health Level 7 protocol (HL7)

\section{Conclusion}

The health sector in Oman has a lot to offer and certainly to gain through the adoption and use of information technology. Enabling technologies in health care can provide efficient access to the much-needed health services. Currently the country's needs in health care are mostly catered for by the Ministry of Health and its affiliated institutions including in particular the Sultan Qaboos University Hospital. Most health care providers in the country are under direct supervision of $\mathrm{MOH}$. This makes it easier in some ways to automate and generalize the health services. Although it may not be fully utilized, Al-Shifa system is in prominent use in all health centers in Oman. It still needs and has the potential to be improved in a number of aspects. Coverage and integration being the most needed aspects of improvement. Further developments are needed in areas of legal aspects, confidentiality and ownership. Since the technology is growing very fast in the health sector, and as there are many systems in Oman, we believe that it is the right time to take the necessary steps toward 


\section{REVIEW OF HEALTH INFORMATION SYSTEMS IN OMAN}

integrating these systems. This will improve patient care delivery by reducing health care cost, providing fast and simultaneous access to the same patient record from any hospital in Oman, and enabling the creation and use of a national healthcare database in Oman.

\section{References}

ARDEN SYNTAX. URL: http://cslxinfmtcs.csmc.edu/hl7/arden/

ASBRU. The Asgaard Project. URL: http://www.asgaard.tuwien.ac.at

BEALE, T. 2001. Health Information Standards Manifesto. V2.5, December 2001. URL: http://www.deepthought.com.au/health/HIS manifesto/his manifesto.pdf.

BEALE, T. 2002. Unified EHR Standards - Is convergence possible? Stud Health Technol Inform.87: 78-97.

BEALE, T. and HEARD, S. 2006. openEHR Architecture: Architecture Overview, openEHR Release 1, The openEHR Foundation, September, 2006.

BIRD L., GOODCHILD, L. and Tun, Z. 2003. Experiences with a Two-Level Modeling Approach to Electronic Health Records, the Journal of Research and Practice in Information Technology, 35(2): 121-137.

BOTT, O.J. 2004. The Electronic Health Record: Standardization and Implementation. $2^{\text {nd }}$ OpenECG Workshop, 2004, Berlin, Germany.

CEN/TC 251. European Committee for Standardization - Technical Committee on Health Informatics. URL: http://www.centc251.org/

CHATTY, D. 1983. The Bedouin of Central Oman. Journal of Oman Studies, 6: 149-162.

CHT, IDX Systems Corporation, 2005. Accelerating transformation through health information technology. Washington, DC: Summary of Findings from the CHT Connectivity Conference, October 18, 2005. URL: http://www.healthtransformation.net/

EBELL, M.H., GASPAR, D.L. and KHURANA, S. 1997. Family physicians' preference for computerized decisionsupport hardware and software. The Journal of Family Practice, 45(2): 127-128.

EICHELBERG, M., ADEN, T., RIESMEIR, J., DOGAC, A. and LALECI, G.B., 2005. A Survey and Analysis of Electronic Healthcare Record Standards. ACM Computing Surveys, 37(4): 277-315.

EMRO, 2006. Regional health systems observatory, WHO. URL: http://gis.emro.who.int/HealthSystemObservatory/PDF/Oman/Full\%20Profile.pdf.

ESCWA. 2005. National profile for the information society in Oman, 2005 URL: http://www.escwa.org.lb/wsis/reports/docs/Oman 2005-E.pdf.

FISCHER, S., STEWARD, T.E., MEHTA, S., WAX, R., and LAPINSKY, S.E. 2003. Handheld Computing in Medicine. $J$. of the American Medical Informatics Association, 10: 139-49.

GARDE, S., KNAUP, P., HOVENGA, E. and HEARD, S. 2007. Towards Semantic Interoperability for Electronic Health Records: Domain knowledge Governance for openEHR Archetypes. Methods Inf Med; 46(1): 332-343.

GREIVER, M. 2001. Evidence-based medicine at the palm of your hand. Canadian Medical Association Journal, 164(2): 250.

HANDLER, T., HOLTMEIER, R., METZGER, J., OVERHAGE, M., TAYLOR, S. and UNDERWOOD, C. 2003, HIMSS Electronic Health Record Definitional Model, Health Information Management Systems Society, 2003: 1-8.

HL7, 2004. HL7 EHR System Functional Model: A Major Development Towards Consensus on Electronic Health Record System Functionality, A White Paper, 2004.

HL7. Health Level Seven. URL: http://www.hl7.org, accessed on 29/1/2007.

IHE. Integrating the healthcare enterprise. URL: http://www.ihe.net/

ISO/TC 215. Inter Standards Organization, Health Informatics Technical Committee 215 URL:http://www.iso.org/iso/en/stdsdevelopment/tc/tclist/echnicalCommitteeDetailPage.T echnicalCommitteeDetail? COMMID $=4720$, accessed on 29/1/2007.

KATRHAKIS, D.G. AND TSIKNAKIS, M. 2006. Electronic Health Record. Technical Report. 


\section{MOHAMMED ELHADI, ET AL}

MINISTRY OF HEALTH, 1992. National health Policy Statement, Ministry of Health, Oman (November 1992. URL: http://www.moh.gov.om.

NHR TASK FORCE, Australia., 2002. The health Information Network for Australia. July 2000. URL: http://health.gov.au/healthonline/healthconnect.

OCEAN INFORMATICS, 2007. EHR Standards. URL: http://www.oceaninformatics.biz/standards.html, accessed on 29/1/2007.

OPENEHR PRIMER. URL: http://www.openehr.org/getting_started/t_openehr_primer.htm.

OPENEHR, URL: http://www.openehr.org/

ORTIZ, E., and CLANCY, C. 2003. Use of Information Technology to Improve the Quality of Health Care in United States. Health Services Research. 38(2): xi-xxii, USA .

PROFORMA LANGUAGE FOR SECTION SUPPORT. URL: http://www.acl.icnet.uk/lab/proforma.html.

PRYOR T.A. and HRIPCSAK. G. 1993, The Arden Syntax for medical logic modules. Int J Clin Monit Comput, 10(4): $215-24$.

RIBICK, A. 2006. CEN TC, ISO TC and HL7 Chairs Announce Broad Agreement on Coordination and Collaboration. Health Level Seven, Inc., November, 2006.

RSNA. Radiological Society of North America. URL: http://www.rsna.org/.

SCHLOEFFEL, P. 2004. Current EHR Developments: an Australian and International Perspective - Part 2 , HINZ, September, 2004.

SHAVER, D. 2006. The HL7 Evolution: Comparing HL7 Version 2 to Version 3. NeoTool.

SOWA, J.F. 2000. Knowledge Representation: Logical, Philosophical, and Computational Foundations, Brooks Cole Publishing Co., Pacific Grove, CA.

STEAD, W.W., MILLER, R.A., MUSEN, M.A. and HERSH, W.R. 2000. Integration and beyond: linking information from disparate sources and into workflow. Journal of the American Medical Informatics Association, 7(2): 135-45.

USA HHS. 2004. Harnessing Information Technology to Improve Health Care: Fact sheet. URL: http://www.hhs.gov/news/press/2004pres/20040427a.html.

WHO. 2004. Essential Health Technologies: Strategy 2004-7. URL: http://www.who.int/eht/en/

WILKIE, B. 2005. Regional Profiles: Oman Rapid Progress. Middle East Health Magazine. URL: http://www.middleeasthealthmag.com/sep2005/feature4.htm.

Received 16 February 2007

Accepted 23 October 2007 